\title{
Photophysical properties and estimation of ground and excited state dipole moments of 7-diethylamino and 7-diethylamino-4-methyl coumarin dyes from absorption and emission spectra
}

\author{
Mohd Mudassir Husain, ${ }^{\mathrm{a},}$, Rajeev Sindhu ${ }^{\mathrm{a}, \mathrm{b}}$ and Harmesh Chander Tandon ${ }^{\mathrm{c}}$ \\ a Physics Section, Department of Applied Sciences and Humanities, Faculty of Engineering and Technology, Jamia Millia Islamia, a Central University, New Delhi- \\ 110025, India \\ b New Green Field College of Engineering and Technology, Palwal, Haryana-121102, India \\ c Department of Chemistry, Sri Venketswara College, Delhi University, New Delhi-110021, India \\ ${ }^{*}$ Corresponding author at: Physics Section, Department of Applied Sciences and Humanities, Faculty of Engineering and Technology, Jamia Millia Islamia, a Central

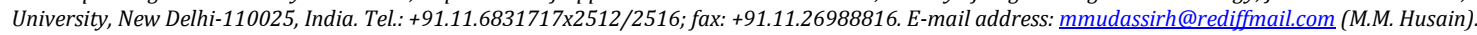

\section{ARTICLE INFORMATION}

Received: 05 September 2011

Received in revised form: 24 October 2011

Accepted: 24 October 2011

Online: 31 March 2012

\section{KEYWORDS}

\section{Coumarins}

Absorption

Stoke's shift

Fluorescence

Dipole moment

Onsager's cavity radius

\section{Introduction}

Coumarins are well known laser dyes [1,2] and are useful probes in different chemical and photochemical studies [3-7]. Most of the coumarins are highly fluorescent and have potential applications as fluorescent indicators [8], sunburn preventives [9], estimation of enzymes etc. [10].

In the present work we have estimated the dipole moments $\left(\mu_{\mathrm{g}}, \mu_{\mathrm{e}}\right)$ and change in dipole moment value $(\Delta \mu)$ of 7-diethyl amino coumarin (7DEAC) and 7-diethylamino-4-methyl coumarin (7DEA4MC). Determination of ground and excited state dipole moments is important, because these values give information about the change in electronic distribution upon excitation. Generally the lifetime of excited states is small and dipole moments of short-lived species are of considerable interest because just as for stable molecules, they provide important information on the electronic and geometrical structures of these transients, furthermore dipole moment represents a direct measure of electron distribution in a molecule of known geometry. A prior knowledge of the dipole moments of the electronically excited species is often useful in the design of non-linear optical materials [11] and in the elucidation of the nature of the excited states. Experimental data on excited states are also useful in the parameterization of semi-empirical quantum chemical procedures for such states.

It is known that the electronic spectra of coumarin dyes are influenced by their immediate environment, among the major environmental factors influencing the electronic spectra,

\begin{abstract}
In the present work, the effect of solvents on absorption and fluorescence spectra and dipole moments $\left(\mu_{\mathrm{g}}, \mu_{\mathrm{e}}\right)$ of 7-diethylamino coumarin (7DEAC) and 7-diethylamino-4-methy intermolecular interactions from non-polar hexane to strongly polar formamide. Using the dipole moment value $(\Delta \mu)$ was also calculated by using the variation of Stoke's shift with results, as well as some of the solvatochromic results that were not studied earlier in such large number of solvents. It is observed that the values of excited singlet-state dipole moments are higher than the ground state ones in both the molecules, which shows that excited states are more polar than the ground states.
\end{abstract}

solvent effects are of particular importance. The change of solvent affects the ground and excited states differently and a systematic analysis of the solvent effect is useful in understanding the excited state behavior of the molecule. The solvent shifts can be accounted in terms of the overall effect of the interaction forces on the $\pi$-electron system of the molecule. It is also known that, as the $\pi$-electron system becomes more delocalized, the transition energy becomes smaller resulting in a bathchromic shift (red shift) and its opposite effect gives rise to a hypsochromic shift (blue shift). In order to assign the electronic transitions as $\pi \rightarrow \pi^{*}$ or $n \rightarrow \pi^{*}$ the solvatochromic technique is found to be very informative. It is known that $\pi \rightarrow \pi^{*}$ bands show a red shift in the solvents of increasing polarity while $n \rightarrow \pi^{*}$ bands show a blue shift [12]. The solvatochromic shifts were also used for the determination of the excited singlet-state dipole moments of some coumarins [13-19].

\section{Experimental}

Coumarin dyes 7DEAC and 7DEA4MC were obtained from Sigma Aldrich Chemicals (USA) and were used as received. The molecular structures of the systems are given in Scheme 1. All the 22 solvents (Formamide, dimethyl sulfoxide, dimethyl formamide, acetonitrile, ethanol, acetone, propanol, butanol, 1pentanol, dichloromethane, ethyl acetate, ethyl benzoate, ethyl propionate, $n$-butyl acetate, chloroform, toluene, $p$-xylene, benzene, carbon tetrachloride, 1,4-dioxane, cyclohexane and 
hexane) used were of spectroscopic grade and were found to be transparent and non-fluorescent in the range of excitation and fluorescence emission. The absorption and fluorescence spectra were measured by Shimadzu-UV-Visible spectrophotometer (UV2450) and Shimadzu spectrofluorometer (RF5301PC), respectively. All the measurements were carried out at room temperature keeping dye concentration low $\left(\sim 10^{-6} \mathrm{M}\right)$ in order to avoid self absorption.

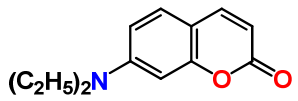

7-diethylamino coumarin (7DEAC)

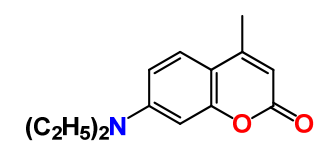

7-diethylamino-4-methyl coumarin (7DEA4MC)

Scheme 1

\section{Theoretical consideration}

In this work we report different solvent parameters e.g. dielectric constant $(\varepsilon)$, refractive index $(n)$ and spectral parameters such as Stoke's shift which is useful for determination of dipole moments. The details of the methods adopted to calculate the dipole moment in ground and excited states of two molecules under consideration based on absorption and fluorescence shifts in various solvents is given below.

\subsection{Determination of dipole moment}

The dipole moment of a molecule in the excited singletstate is determined by the effect of electric field (internal and external) on the position of its spectral band. Two methods depending on the internal electric field (solvatochromism) have been employed in the present investigation.

\subsubsection{Method I}

By employing the simplest quantum-mechanical second order perturbation theory and taking into account Onsager's model, Bilot and Kawski $[20,21]$ have obtained an expression for the spectral shift given by

$$
\begin{aligned}
& \Delta v_{a, f}=-m_{a, f}\left(1-\alpha f^{\prime}\right)^{-1}\left[f(1-\alpha f)^{-1}-f^{\prime}\left(1-\alpha f^{\prime}\right)^{-1}\right] \\
& -\frac{\mu_{e}^{2}-\mu_{g}^{2}}{2 h c}\left(2-\alpha f^{\prime}\right) f^{\prime}\left(1-\alpha f^{\prime}\right)^{-2}
\end{aligned}
$$

where

$$
m_{a}=\frac{\mu_{\mathrm{g}}\left(\mu_{e}-\mu_{g}\right)}{h c}
$$

for absorption and

$$
m_{f}=\frac{\mu_{\mathrm{e}}\left(\mu_{e}-\mu_{g}\right)}{h c}
$$

for fluorescence $\alpha$ is the mean static isotropic polarizability of the solute. $f$ and $f^{\prime}$ are the reaction field factors which depend on the shape and the Onsager's cavity radius $a$ of the solute, and on the relative permittivity $(\varepsilon)$ and refractive index $(n)$ of the solvent. $v_{a}$ and $v_{f}$ are the absorption and fluorescence maxima and $h$ and $c$ are Planck's constant and velocity of light in vacuum, respectively.

Based on the Equation 1, the following expressions are obtained for $\left(v_{a}-v_{f}\right)$ and $\left(v_{a}+v_{f}\right) / 2[21,22]$;

$v_{a}-v_{f}=m_{1} f(\varepsilon, n)+$ constant

$\frac{1}{2}\left(v_{a}+v_{f}\right)=-m_{2} \varphi(\varepsilon, n)+$ constant

where

$\varphi(\varepsilon, n)=\frac{1}{2} f(\varepsilon, n)+g(n)$

$m_{1}=\frac{2\left(\mu_{e}-\mu_{g}\right)^{2}}{h c a^{3}}$

and

$m_{2}=\frac{2\left(\mu_{\mathrm{e}}^{2}-\mu_{\mathrm{g}}^{2}\right)}{h c a^{3}}$

The factors $f$ and $f^{\prime}$ are simplified when a spherical cavity of radius $a$ is assumed, which may be regarded as a sufficient approximation [22]. In this case, solvent polarity parameters $f$ $(\varepsilon, n)$ and $g(n)$ have the form

$f(\varepsilon, n)=\frac{((\varepsilon-1) /(2 \varepsilon+1))-\left(\left(n^{2}-1\right) /\left(2 n^{2}+1\right)\right)}{\left(\left(1-\left(2 \alpha / a^{3}\right)\right)((\varepsilon-1) /(2 \varepsilon+1))\left(\left(1-\left(2 \alpha / a^{3}\right)\right)\left(n^{2}-1\right) /\left(2 n^{2}+1\right)\right)\right.}$

$g(n)=\frac{\left(\left(n^{2}-1\right) /\left(2 n^{2}+1\right)\right)\left(\left(1-\left(\alpha / a^{3}\right)\right)\left(\left(n^{2}-1\right) /\left(2 n^{2}+\right)\right)\right.}{\left(1-\left(2 \alpha / a^{3}\right)\right)\left(\left(n^{2}-1\right) /\left(2 n^{2}+1\right)\right)}$

If the polarizability of the solute is neglected, i.e. $\alpha=0$, then Equation 9 leads to Lippert-Mataga relation [23-26]

$F(\varepsilon, n)=\left[\frac{\varepsilon-1}{2 \varepsilon+1}-\frac{n^{2}-1}{2 n^{2}+1}\right]$

It is based on the Onsager's reaction field theory, which assumes that the fluorophore is a point dipole residing in the center of a spherical cavity with radius in a homogeneous and isotropic dielectric with relative permittivity $\varepsilon$. The LippertMataga equation breaks down when in addition to the nonspecific interactions, specific fluorophore-solvent interactions e.g., hydrogen bonding, electron-pair donor/electron-pair acceptor interactions also contribute significantly to the solutesolvent interaction. Another limitation results from the cavity radius, which is not easy to estimate for non-spherical molecule.

For an isotropic polarizability of the solute, the condition $2 \alpha / a^{3}=1$ is frequently satisfied and justified [22], and functions $f(\varepsilon, n)$ and $\varphi(n)$ leads to Bakhshiev and Kawski-ChammaViallet relations, respectively $[27,28]$

$F_{1}(\varepsilon, n)=\frac{2 n^{2}+1}{n^{2}+2}\left[\frac{\varepsilon-1}{\varepsilon+2}-\frac{n^{2}-1}{n^{2}+2}\right]$

$F_{2}(\varepsilon, n)=\frac{1}{2} F_{1}+\frac{3}{2} \frac{\left(n^{4}-1\right)}{\left(n^{2}+2\right)^{2}}$ 
According to McRae theory $[29,30] f(\varepsilon, n)$ can be written as

$F_{3}(\varepsilon, n)=\left[\frac{\varepsilon-1}{\varepsilon+2}-\frac{n^{2}-1}{n^{2}+2}\right]$

Using Equations 7 and 8, the values of $\mu_{\mathrm{g}}$ and $\mu_{\mathrm{e}}$ can be obtained as;

$$
\begin{aligned}
& \mu_{\mathrm{g}}=\frac{m_{2}-m_{1}}{2} \sqrt{\frac{h c a^{3}}{2 m_{1}}} \\
& \mu_{\mathrm{e}}=\frac{m_{1}+m_{2}}{2} \sqrt{\frac{h c a^{3}}{2 m_{1}}}
\end{aligned}
$$

or

$$
\mu_{e}=\left[\frac{m_{1}+m_{2}}{m_{2}-m_{1}}\right] \mu_{g} ; m_{2}>m_{1}
$$

So the ratio of dipole moments in excited state and ground state is given by

$$
\frac{\mu_{e}}{\mu_{g}}=\frac{m_{1}+m_{2}}{m_{2}-m_{1}}
$$

The parameters $m_{1}$ and $m_{2}$ occurring for the differences $\left(v_{a^{-}}\right.$ $\left.v_{f}\right)$ and the sum $\left(v_{a}+v_{f}\right) / 2$ of the wave numbers are linear functions of the solvent polarity parameters $F_{1}(\varepsilon, n)$ and $F_{2}(\varepsilon, n)$ and can be determined from the slopes of the straight lines.

\subsubsection{Method II (Molecular-microscopic solvent polarity $\operatorname{parameter}\left(E_{T}^{N}\right)$}

The second method is based on the empirical polarity scale proposed by Reichardt [31] and gives results with solvatochromic shift of dipolar molecules that correlates much better with molecular-microscopic solvent polarity parameter $E_{T}^{N}$ rather than the traditionally used bulk solvent polarity functions as in the later the error estimation of Onsager cavity radius ' $a$ ' has been minimized. The theoretical basis for the correlation as spectral shift with $E_{T}^{N}$ has been developed by Ravi et al. [32] and accordingly, the excited state dipole moment is evaluated by equation

$v_{a}-v_{f}=11307.6\left[\left(\frac{\Delta \mu}{\Delta \mu_{\mathrm{B}}}\right)^{2}\left(\frac{a_{B}}{a}\right)^{3}\right] E_{T}^{N}+$ constant

$E_{T}^{N}$ [or equivalently, $\left.E_{T}(30)\right]$ is the solvent polarity function proposed by Reichardt, based on the absorption wave number, $v_{a}$, of a standard Betaine dye in the solvent. It is expressed as

$E_{T}^{N}=\frac{E_{T}(\text { solvent })-E_{T}(\mathrm{TMS})}{E_{T}(\text { water })-E_{T}(\mathrm{TMS})}$

Here $E_{T}(30)$ value for a solvent is simply defined as molar transition energy of the dissolved betaine dye measured in $\mathrm{kcal} / \mathrm{mol}$ for the charge transfer absorption band $\left(v_{a}\right)$ and is expressed as

$$
E_{T}(30)\left[\mathrm{kcal}-\mathrm{mol}^{-1}\right]=2.8591 \times 10^{-3} v_{a}\left[\mathrm{~cm}^{-1}\right]
$$

$\Delta \mu_{\mathrm{B}}$ and $a_{\mathrm{B}}$ are the dipole moment changes on excitation and the Onsager radius respectively, of Betaine dye. $\Delta \mu$ and $a$ are the corresponding quantities of the molecule of interest. Equation 19 clearly illustrates that the Stokes shift $\left(v_{a}-v_{f}\right)$ changes linearly with the solvent polarity function $E_{T}^{N} . \Delta \mu$ can be obtained from the slope of Equation 19. Using the reported $\Delta \mu_{\mathrm{B}}=9 \mathrm{D}$ of Betaine dye and its Onsager radius $6.2 \AA$ [33], the change in dipole moment is determined by

$$
\Delta \mu=\mu_{\mathrm{e}}-\mu_{\mathrm{g}}=\sqrt{\frac{m \times 81}{11307.6 \times\left(\frac{6.2}{a}\right)^{3}}}
$$

In the present work, the Onsager's cavity radii of the dye molecules were taken as half the distance between the amino $\mathrm{N}$ atom and the carbonyl $\mathrm{O}$ atom of the systems, optimized by semiempirical models (AM1 and PM3). This is consistent with the fact that these atoms provide the strongest dipole vector component in all the molecules considered [33]. In addition to this we have also calculated the Onsager's cavity radius by the formula [34]

$a=\sqrt[3]{\frac{3 \mathrm{M}}{4 \pi \rho \mathrm{N}}}$

where $\mathrm{M}$ is the molecular weight of the dye; $\rho$ is the density of the dye; $\mathrm{N}$ is the Avogadro's number. It is difficult to calculate the density of a compound and the density of the compounds are not listed in literatures so we have approximated [35] the densities of these dyes to be $1 \mathrm{~g} / \mathrm{cm}^{-3}$.

\section{Results and discussion}

Absorption and fluorescence spectra of 7DEAC and 7DEA4MC were measured in solvents of different solvent parameters e.g., refractive index and dielectric constant. Estimation of ground state and excited state dipole moments are done experimentally from observed absorption and emission spectra of dye molecules in various polar and nonpolar solvents. The photophysical parameters; absorption and emission maxima wave numbers $\left(v_{a}, v_{f}\right)$, Stoke's shift $\left(v_{a}-v_{f}\right)$ and arithmetic mean of Stoke's shift $\left(v_{a}+v_{f}\right) / 2$ values (in $\left.\mathrm{cm}^{-1}\right)$ for both the coumarin dyes in different solvents are tabulated in Table 1. It has been observed that the emission peaks are more pronounced than the absorption peaks for both the molecules with increasing solvent polarity which gives a larger spectral shift in the emission spectra than in the absorption spectra of the molecules. With increase in the polarity of the solvent, the fluorescence emission peak undergoes a bathochromic shift (red shift), confirming a $\pi \rightarrow \pi^{*}$ transition [30]. The less pronounced shift in the absorption spectra observed in all the solvents indicates that the ground-state energy distribution is not affected to a greater extent possibly due to the less polar nature of the dyes in the ground state than in the excited state which further indicates that of $\mu_{\mathrm{e}}>\mu_{\mathrm{g}}$. The red shift of the fluorescence wavelengths could be due to the marked difference between the excited-state charge distribution in the solute and the ground-state charge distribution, resulting in a stronger interaction with polar solvents in the excited state.

The values of solvent polarity function $F(\varepsilon, n), F_{1}(\varepsilon, n), F_{2}(\varepsilon$, $n), F_{3}(\varepsilon, n)$ and $E_{T}^{N}$ for solvents used in this work are presented in Table 2. Using linear fit method, the graphs of $\left(v_{a}-v_{f}\right)$ and $\left(v_{a}+v_{f}\right) / 2$ are plotted against solvent polarity functions $F(\varepsilon, n)$, $F_{1}(\varepsilon, n), F_{2}(\varepsilon, n)$ and $E_{T}^{N}$, respectively for 7DEAC (Figures 1-4). The slopes, intercepts and correlation coefficients of these best fit lines are given in Table 3. Figure $5 \mathrm{a}$ shows the solvatochromic shifts of the absorption spectra of 7DEAC in different solvents at room temperature. 
Table 1. Spectral data of 7DEAC and 7DEA4MC in various solvents.

\begin{tabular}{|c|c|c|c|c|c|c|c|c|}
\hline \multirow[t]{2}{*}{ Solvents } & \multicolumn{4}{|c|}{ 7DEAC } & \multicolumn{4}{|c|}{ 7DEA4MC } \\
\hline & $v_{a}\left(\mathrm{~cm}^{-1}\right)$ & $v_{f}\left(\mathrm{~cm}^{-1}\right)$ & $v_{a}-v_{f}\left(\mathrm{~cm}^{-1}\right)$ & $\left(v_{a}+v_{f}\right) / 2\left(\mathrm{~cm}^{-1}\right)$ & $\nu_{a}\left(\mathrm{~cm}^{-1}\right)$ & $v_{f}\left(\mathrm{~cm}^{-1}\right)$ & $v_{a}-v_{f}\left(\mathrm{~cm}^{-1}\right)$ & $\left(v_{a}+v_{f}\right) / 2\left(\mathrm{~cm}^{-1}\right)$ \\
\hline Formamide & 25906 & 21598 & 4308 & 23752 & 26267 & 21978 & 4289 & 24122 \\
\hline DMSO & 26315 & 22321 & 3994 & 24318 & 26680 & 22727 & 3953 & 24703 \\
\hline Dimethyl formamide & 26525 & 22371 & 4154 & 24448 & 27027 & 22988 & 4039 & 25007 \\
\hline Acetonitrile & 26737 & 22301 & 4436 & 24519 & 27107 & 22946 & 4161 & 25026 \\
\hline Ethanol & 26490 & 21978 & 4512 & 24234 & 26780 & 22271 & 4509 & 24525 \\
\hline Acetone & 26881 & 22988 & 3893 & 24934 & 27322 & 23386 & 3936 & 25354 \\
\hline Propanol & 26455 & 22172 & 4283 & 24313 & 26702 & 22371 & 4331 & 24536 \\
\hline Butanol & 26455 & 22123 & 4332 & 24289 & 26716 & 22471 & 4245 & 24593 \\
\hline 1-Pentanol & 26525 & 22172 & 4353 & 24348 & 26737 & 22471 & 4266 & 24604 \\
\hline Dichloromethane & 26525 & 22914 & 3611 & 24719 & 26867 & 23485 & 3382 & 25176 \\
\hline Ethyl Acetate & 27277 & 23752 & 3525 & 25514 & 27578 & 23877 & 3701 & 25727 \\
\hline Ethyl Benzoate & 26737 & 23326 & 3411 & 25031 & 27173 & 23696 & 3477 & 25434 \\
\hline Ethyl Propionate & 27322 & 23518 & 3804 & 25420 & 27777 & 23866 & 3911 & 25821 \\
\hline n-Butyl Acetate & 27472 & 23980 & 3492 & 25726 & 27793 & 23980 & 3813 & 25886 \\
\hline Chloroform & 26385 & 22935 & 3450 & 24660 & 26723 & 23255 & 3468 & 24989 \\
\hline Toluene & 27442 & 23866 & 3576 & 25654 & 27855 & 24213 & 3642 & 26034 \\
\hline$p$-Xylene & 27397 & 23529 & 3868 & 25463 & 27940 & 24319 & 3621 & 26129 \\
\hline Benzene & 27247 & 23724 & 3523 & 25485 & 27777 & 24154 & 3623 & 25965 \\
\hline Carbon tetrachloride & 27677 & 24131 & 3546 & 25904 & 28066 & 24691 & 3375 & 26378 \\
\hline 1,4-Dioxane & 27397 & 23685 & 3712 & 25541 & 27700 & 23752 & 3948 & 25726 \\
\hline Cyclohexane & 28089 & 25087 & 3002 & 26588 & 28506 & 25380 & 3126 & 26943 \\
\hline Hexane & 28248 & 25316 & 2932 & 26782 & 28636 & 25641 & 2995 & 27138 \\
\hline
\end{tabular}

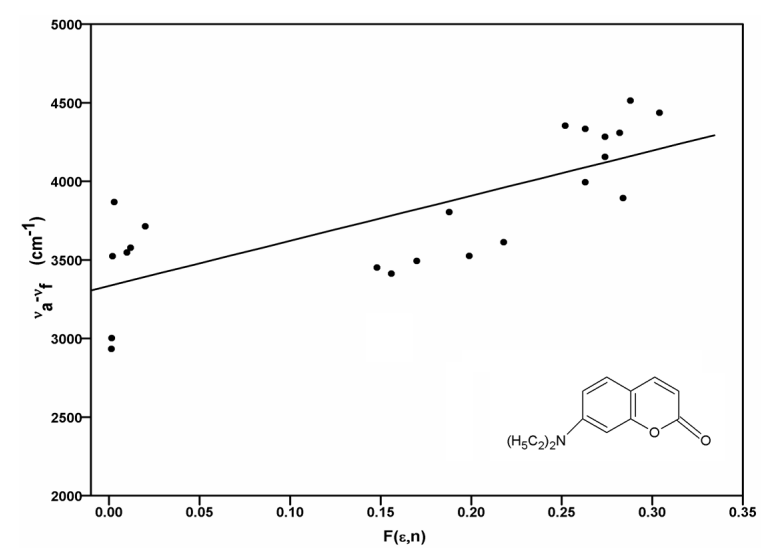

Figure 1. Plot of Stoke's shift $v_{a}-v_{f}\left(\mathrm{~cm}^{-1}\right)$ versus $F(\varepsilon, n)$ of 7DEAC.

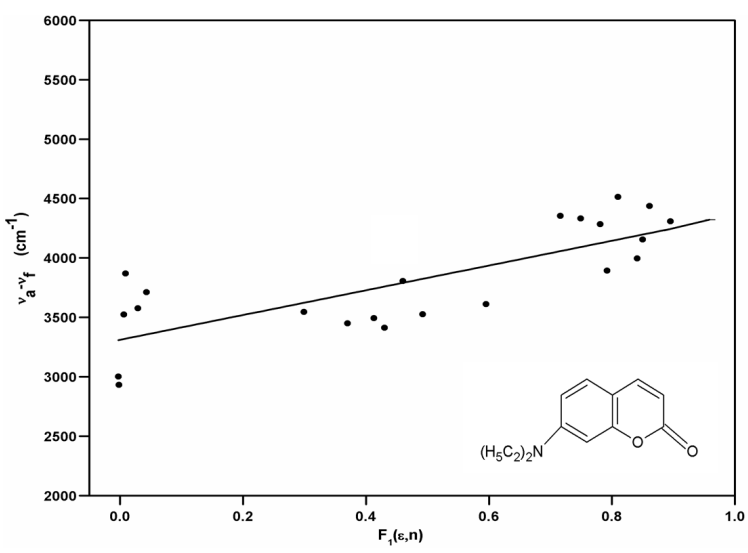

Figure 2. Plot of Stoke's shift $v_{a}-v_{f}\left(\mathrm{~cm}^{-1}\right)$ versus $F_{1}(\varepsilon, n)$ of 7DEAC.

Figure $5 b$ shows a typical example of the solvent effect on the electronic emission spectrum of 7DEAC. The geometry of these systems was optimized using semi empirical methods AM1, PM3 to obtain the Onsager's cavity radius. The values of Onsager's cavity radius obtained by these methods and by Equation 23 along with the calculated ground $\left(\mu_{\mathrm{g}}\right)$ and singlet excited-state $\left(\mu_{\mathrm{e}}\right)$ dipole moments and the ratio $\left(\mu_{\mathrm{e}} / \mu_{\mathrm{g}}\right)$ for both the coumarin dyes estimated by using Equations 15, 16 and 17 are given in Table 4 . The difference $(\Delta \mu)$ in excited and groundstate dipole moments calculated by using method employed by Lippert-Mataga, Bakhshiev, McRae and molecular-microscopic based on solvent polarity relations are also given in the same table. It can be seen that the $\Delta \mu$ values obtained for both the molecules by Lippert-Mataga method are large as compared to values obtained by all other methods, it is due to the fact that this method does not take into account the polarizability of the solute. A comparison of results obtained in this work with the work reported previously is shown in Table 5.

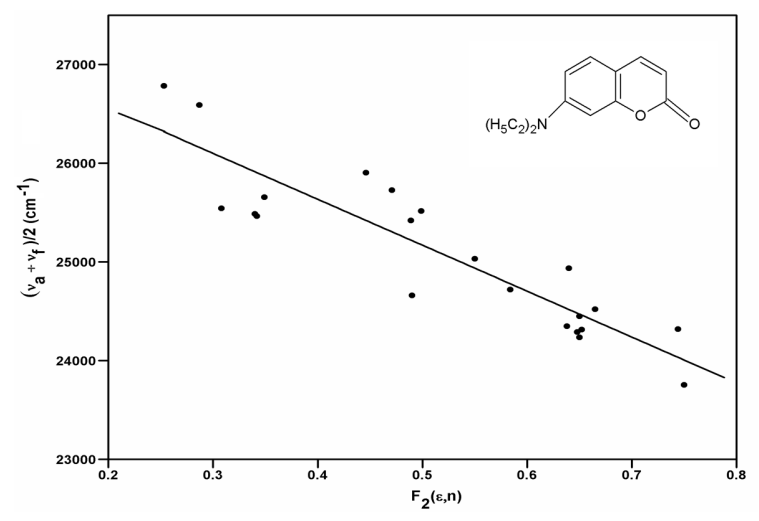

Figure 3. Plot of $\left(v_{a}+v_{f}\right) / 2\left(\mathrm{~cm}^{-1}\right)$ versus $F_{2}(\varepsilon, n)$ of 7DEAC.

The values for the 7DEA4MC in ground and excited states are in the range of $3.48 \mathrm{D}-6.00 \mathrm{D}$ and $5.13 \mathrm{D}-8.85 \mathrm{D}$ respectively. Earlier, M. Diraisan et al. [36] reported the computed value as $\mu_{\mathrm{g}}=8.05 \mathrm{D}$ and $\mu_{\mathrm{e}}=12.92 \mathrm{D}$ for the said coumarin. The findings of McCarthy and Blanchard [37] shows $\mu_{\mathrm{g}}=6.35 \mathrm{D}$ and $\mu_{\mathrm{e}}=9.81 \mathrm{D}$ which are in good agreement with our experimental results. This inconsistency of dipole moments with these theoretical work arises because, while computing the parameters the molecule is considered as isolated system (as in gas phase), whereas the experimentally obtained values are in solution phase, where the solvent (matrix) introduces strong perturbation. Further it is evident from Table 4 that in both dye molecules, under investigation, the changes in the dipole moments on electronic excitation is rather small. 
Table 2. Refractive index, dielectric constant and various solvent polarity functions of the solvents a.

\begin{tabular}{|c|c|c|c|c|c|c|c|}
\hline Solvents & $n^{\mathbf{b}}$ & $\varepsilon^{\mathbf{b}}$ & $F(\varepsilon, n)$ & $F_{1}(\varepsilon, n)$ & $F_{2}(\varepsilon, n)$ & $F_{3}(\varepsilon, n)$ & $\left(E_{T}^{N}\right)^{\mathbf{b}}$ \\
\hline Formamide & 1.447 & 111.0 & 0.282 & 0.895 & 0.750 & 0.706 & 0.799 \\
\hline DMSO & 1.479 & 47.24 & 0.263 & 0.841 & 0.744 & 0.655 & 0.441 \\
\hline Dimethyl formamide & 1.426 & 37.0 & 0.274 & 0.850 & 0.650 & 0.664 & 0.404 \\
\hline Acetonitrile & 1.344 & 36.64 & 0.304 & 0.861 & 0.665 & 0.710 & 0.472 \\
\hline Ethanol & 1.361 & 24.30 & 0.288 & 0.810 & 0.650 & 0.664 & 0.654 \\
\hline Acetone & 1.359 & 21.01 & 0.284 & 0.792 & 0.640 & 0.649 & 0.355 \\
\hline Propanol & 1.385 & 20.6 & 0.274 & 0.781 & 0.652 & 0.632 & 0.617 \\
\hline Butanol & 1.399 & 17.40 & 0.263 & 0.749 & 0.648 & 0.603 & 0.601 \\
\hline 1-Pentanol & 1.410 & 14.80 & 0.252 & 0.716 & 0.638 & 0.573 & 0.503 \\
\hline Dichloromethane & 1.424 & 8.93 & 0.218 & 0.595 & 0.584 & 0.474 & 0.320 \\
\hline Ethyl Acetate & 1.372 & 6.08 & 0.199 & 0.492 & 0.499 & 0.398 & 0.228 \\
\hline Ethyl Benzoate & 1.503 & 5.99 & 0.156 & 0.430 & 0.550 & 0.328 & - \\
\hline Ethyl Propionate & 1.380 & 5.58 & 0.188 & 0.460 & 0.489 & 0.372 & - \\
\hline$n$-Butyl Acetate & 1.394 & 5.00 & 0.170 & 0.413 & 0.471 & 0.427 & - \\
\hline Chloroform & 1.442 & 4.81 & 0.148 & 0.370 & 0.490 & 0.292 & 0.259 \\
\hline Toluene & 1.497 & 2.38 & 0.012 & 0.029 & 0.349 & 0.020 & 0.099 \\
\hline$p$-Xylene & 1.496 & 2.27 & 0.003 & 0.009 & 0.342 & 0.005 & 0.077 \\
\hline Benzene & 1.501 & 2.28 & 0.002 & 0.006 & 0.340 & 0.004 & 0.117 \\
\hline Carbon tetrachloride & 1.459 & 2.24 & 0.010 & 0.299 & 0.446 & 0.016 & 0.055 \\
\hline 1,4-Dioxane & 1.422 & 2.22 & 0.021 & 0.043 & 0.308 & 0.034 & 0.164 \\
\hline Cyclohexane & 1.426 & 2.02 & 0.0015 & -0.003 & 0.287 & 0.0024 & 0.015 \\
\hline Hexane & 1.374 & 1.88 & 0.0014 & -0.002 & 0.253 & 0.0021 & 0.006 \\
\hline
\end{tabular}

a $F(\varepsilon, n)$ : Lippert, $F_{1}(\varepsilon, n)$ : Bakhshiev, $F_{2}(\varepsilon, n)$ : Kawski-Chamma-Viallet and $F_{3}(\varepsilon, n)$ : McRae's solvent polarity function.

b The values of $n, \varepsilon$ and $E_{T}^{N}$ (Molecular-microscopic solvent function) are from Ref. [31].

Table 3. Slopes and intercepts of linear fittings.

\begin{tabular}{|c|c|c|c|c|}
\hline Molecule & Slope & Intercept & No. of data & Correlation coefficient \\
\hline \multicolumn{5}{|c|}{ Lippert-Mataga correlation } \\
\hline 7DEAC & 2867.56 & 3334.39 & 22 & 0.76 \\
\hline 7DEA4MC & 2559.68 & 3394.41 & 22 & 0.74 \\
\hline \multicolumn{5}{|c|}{ Bakhshiev correlation } \\
\hline 7DEAC & 1042.18 & 3311.25 & 22 & 0.64 \\
\hline 7DEA4MC & 898.17 & 3387.67 & 22 & 0.59 \\
\hline \multicolumn{5}{|c|}{ Kawski-Chamma-Viallet correlation } \\
\hline 7DEAC & 4654.22 & 27496.18 & 22 & 0.81 \\
\hline 7DEA4MC & 4679.78 & 27881.08 & 22 & 0.79 \\
\hline \multicolumn{5}{|c|}{ McRae correlation } \\
\hline 7DEAC & 1234.33 & 3343.44 & 22 & 0.77 \\
\hline 7DEA4MC & 1103.07 & 3402.01 & 22 & 0.76 \\
\hline \multicolumn{5}{|c|}{$E_{T}^{N}$ correlation } \\
\hline 7DEAC & 1698.68 & 3289.77 & 19 & 0.74 \\
\hline 7DEA4MC & 1576.36 & 3309.68 & 19 & 0.75 \\
\hline
\end{tabular}

Table 4. Ground and excited-state dipole moments (Debye) and Onsager radius ( $\AA$ ) of 7DEAC and 7DEA4MC.

\begin{tabular}{|c|c|c|c|c|c|c|c|c|}
\hline Molecule & Onsager radius, $a$ & $\mu_{\mathrm{ga}}$ & $\mu_{\mathrm{e}^{\mathrm{b}}}$ & $\Delta \mu \mathrm{c}$ & $\Delta \mu \mathrm{d}$ & $\Delta \mu \mathrm{f}$ & $\Delta \mu \mathrm{g}$ & $\left(\mu_{\mathrm{e}} / \mu_{\mathrm{g}}\right)_{\mathrm{h}}$ \\
\hline \multirow{3}{*}{ 7DEAC } & 3.50(AM1) & 3.65 & 5.75 & 2.10 & 3.49 & 2.29 & 1.47 & 1.58 \\
\hline & 3.47(РM3) & 3.61 & 5.68 & 2.09 & 3.44 & 2.26 & 1.46 & 1.58 \\
\hline & 4.41(Eq.19) & 5.16 & 8.14 & 2.98 & 5.08 & 3.23 & 2.09 & 1.58 \\
\hline \multirow{3}{*}{ 7DEA4MC } & 3.13(AM1) & 3.48 & 5.13 & 1.65 & 2.79 & 1.83 & 1.20 & 1.47 \\
\hline & $3.45(\mathrm{PM} 3)$ & 4.05 & 5.94 & 1.89 & 3.22 & 2.11 & 1.40 & 1.47 \\
\hline & 4.50(Eq.19) & 6.00 & 8.85 & 2.85 & 4.8 & 3.15 & 2.07 & 1.48 \\
\hline
\end{tabular}

a Ground state dipole moments calculated from Equation 15.

b Excited state dipole moments calculated from Equation 16.

c $\Delta \mu$ calculated from Bakhshiev model.

d $\Delta \mu$ calculated from Lippert-Mataga model.

f $\Delta \mu$ calculated from McRae model.

g $\Delta \mu$ calculated from molecular-microscopic solvent function $\left(E_{T}^{N}\right)$.

$\mathrm{h}$ The ratio of excited state and ground state dipole moments calculated from Equation 17.

This suggests that the emission of these dyes originates from a state which although more polar than ground state is probably a locally excited intramolecular-charge transfer (ICT) state. Charge transfer accompanying excitation to lowest excited singlet state usually results in the excited molecule having a greater dipole moment than the ground state [38].The longest absorption and fluorescence maxima shifted to the lower energy end of the spectrum on increasing the solvent polarity for coumarin molecule. It is known that the lowest excited state is $n \rightarrow \pi^{*}$, but the substituted coumarin, the energy difference between $n \rightarrow \pi^{*}$ and $\pi \rightarrow \pi^{*}$ levels becomes smaller with increasing substitution and in some cases even the state order gets inverted [39]. In the present case, there is a bathochromic shift on increasing the solvent polarity which indicates that the transition involved is a $\pi \rightarrow \pi^{*}$ transition and lowest lying state is $\pi \rightarrow \pi^{*}$ (see Table 1). The difference between the values of ground-state dipole moment $(\mu \mathrm{g})$ and excited-state dipole moment $\mu_{\mathrm{e}}$ of 7DEAC and 7DEA4MC is small, this could be explained as, a $\mathrm{CH}_{3}$ group at position- 4 has small influence on the energy levels of molecules [38]. The effect of amino group in case of 7DEAC and 7DEA4MC is considered where unshared pair of electrons on this group resides in molecular orbitals (largely localized to amino group). 
Table 5. Comparison of present results with the values reported earlier*

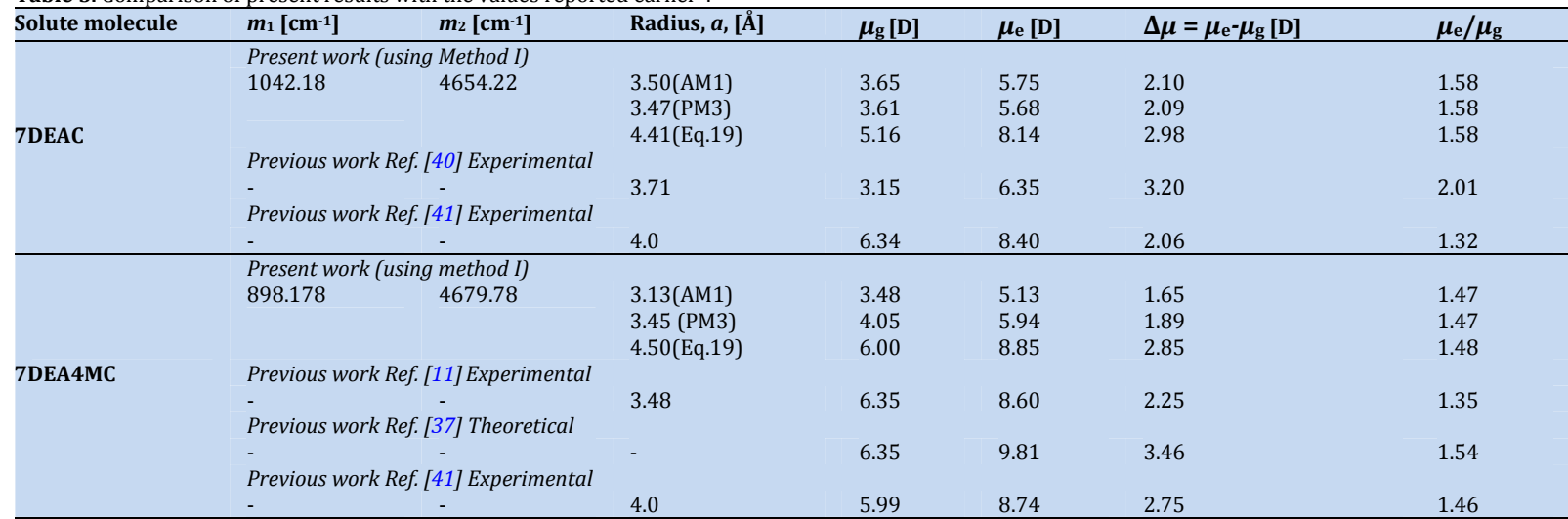

${ }^{*} m_{1}, m_{2}$ are the slopes of $v_{a}-v_{f}$ vs. $F_{1}(\varepsilon, n)$ and $\left(v_{a}+v_{f}\right) / 2$ vs. $F_{2}(\varepsilon, n)$; ' $a$ ' is the Onsager radius; $\mu_{\mathrm{g},} \mu_{\mathrm{e}}$ are the dipole moments in ground and excited state, respectively. "-": Values of $m_{1}$ and $m_{2}$ are not reported in ref. [11], ref. [40], ref. [37] and ref. [41].

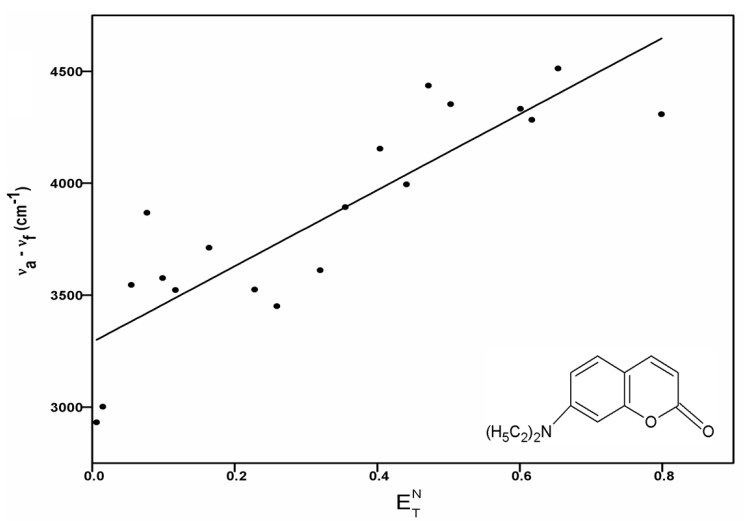

Figure 4. Plot of Stoke's shift $v_{a}-v_{f}\left(\mathrm{~cm}^{-1}\right)$ versus $E_{T}^{N}$ of 7DEAC.

Thus due to ICT the electronic charge from these functional groups gets substantially delocalized throughout the system. Hence, the energy gap between the highest occupied and lowest unoccupied orbitals of amino substituted molecule is considerably lower than the difference between the highest occupied and lowest unoccupied orbitals of unsubstituted molecules $[12,38]$.

\section{Conclusion}

The present investigations of the photophysical properties of 7DEAC and 7DEA4MC, which are promising active medium for tunable lasers, show that the lowest lying excited state of the molecules is $\pi \rightarrow \pi^{*}$. The dipole moments of the molecules are higher in the excited state and it is about 1.6 times of the ground state value. Further, dipole moment values for the coumarin dyes differ from each other. This can be attributed to the structural difference between the molecules. The relative positions of $\pi \rightarrow \pi^{*}$ and $n \rightarrow \pi^{*}$ depend on the nature of the substituent as well as the solvents. The assumption $2 \alpha / a^{3}=1$ is justified and does not significantly influence the determined value of $\mu_{\mathrm{e}}$, because in most cases $\alpha / a^{3}$ is unknown [22]. Equation 17 can be used to estimate the value of the excitedstate dipole moment by pre-knowledge of the value of groundstate dipole moment, without the necessity of knowing the Onsager radius of the solute. Further the absorption and fluorescence spectra of the coumarins are studied in 22 different polar and non-polar solvents which were limited to a few solvents in the earlier reported work.

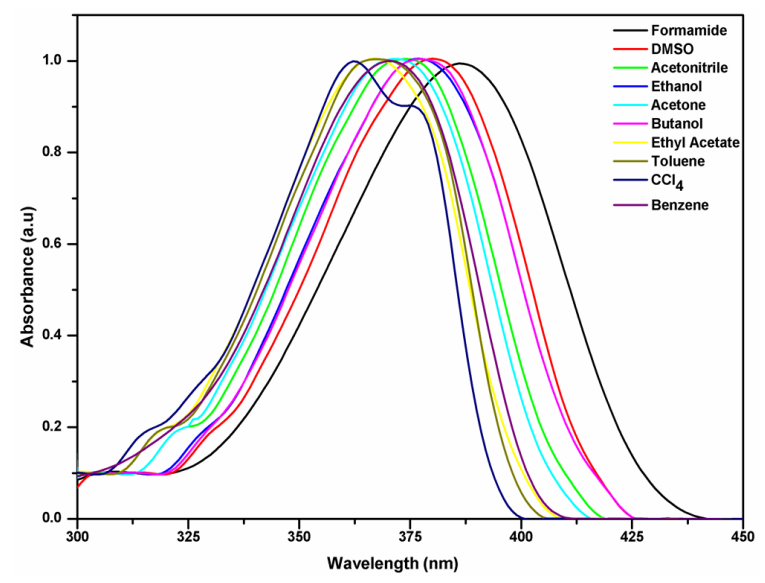

(a)

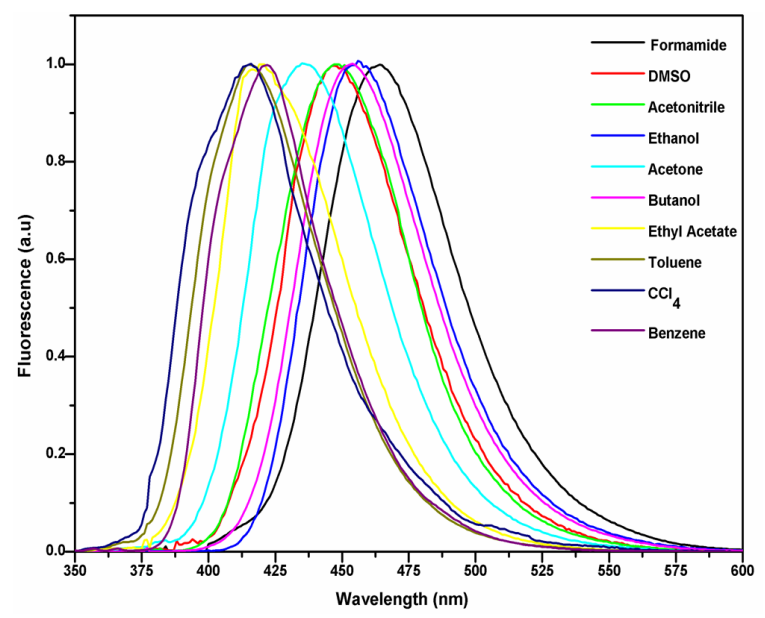

(b)

Figure 5. Absorption (a) and fluorescence (b) spectra of 7DEAC in different solvents.

\section{Acknowledgements}

This work is supported by University Grant Commission through a research grant (F. No. 36-360/2008) provided to one of the authors (Mohammed Mudassir Husain) and Department of Applied Sciences and Humanities, Faculty of Engineering and Technology, Jamia Millia Islamia, New Delhi, India. 


\section{References}

[1]. Fletcher, A. N.; Bliss, D. E. Appl. Phys. 1978, 16, 289-295.

[2]. Halstead, J. A.; Reeves, R. R. Opt. Commun. 1978, 27, 273-276.

[3]. Gardecki, J. A.; Maroncelli, M. J. Phys. Chem. 1999, 103, 1187-1197.

[4]. Levinger, N. E. Curr. Opin. Colloid Interf. Sci. 2000, 5, 118-124.

[5]. Castner Jr., E. W.; Kennedy, D.; Cave, R. J. J. Phys. Chem. A 2000, 104, 2869-2885.

[6]. Morandeira, A.; Fulrstenberg, A.; Vauthey, E. J. Phys. Chem. A 2004, 108, 8190-8200.

[7]. Kumbhakar, M.; Nath, S.; Mukherjee, T.; Pal, H. J. Chem. Phys. 2004, 121, 6026-6033.

[8]. Schitschek, E. J.; Trias, J. A.; Hammond, P. R.; Henry, R. A.; Atkins, R. L. Opt. Commun. 1976, 16, 313-316.

[9]. Parkyani, C.; Antonius, M. S.; Aaron, J. J.; Buna, M.; Tine, A.; Cisse L. Spectrosc. Lett. 1994, 27, 439-449.

[10]. Shermon, W. R.; Robins, E. Anal. Chem. 1968, 40, 803-805.

[11]. Ravi, M.; Soujanya, T.; Samanta. A.; Radhakrishnan, T. P. J. Chem. Soc. Faraday Trans. 1995, 91, 2739-2742.

[12]. Mannekutla, J. R.; Mulimani, B. G.; Inamdar, S. R. Spectochim. Acta A 2008, 69, 419-426.

[13]. Zakerhamidi, M. S.; Ghanadzadeh, A.; Moghadam, M. Spectrochim. Acta A 2011, 78, 961-966.

[14]. Inamdar, S. R.; Nadaf, Y. F.; Mulimani, B. G. J. Mol. Struct. 2003, 624, 47-51.

[15]. Kumar, S.; Rao, V. C.; Rastogi, R. C. Spectrochim. Acta Part A 2001, 57, 41-47.

[16]. Cremers, D. A.; Windsor, M. W. Chem. Phys. Lett. 1980, 71, 27-32.

[17]. Balu, W.; Reber, R.; Penzkofer, A. Opt. Commun. 1982, 43, 210-214.

[18]. Sundstrom, V.; Gillbro, T.; Bergstrom, H. Chem. Phys. 1982, 73, 439458.

[19]. Sundstrom, V.; Gilbro, T. Chem. Phys. Lett. 1984, 109, 538-543.

[20]. Bilot, L.; Kawski, A. Z. Naturforsch. 1962, 179, 621-627

[21]. Kawski, A.; Rabek, J. F. Progress in photochemistry and photophysics, Vol. 5., Boca Raton USA, CRC Press, 1992.

[22]. Kawski, A. Z. Natureforsch. 2002, 57A, 255-262.

[23]. Lippert, E. Z. Naturforch, Part A 1955, 10, 541-545.

[24]. Lippert, E. Elektrochem, Ber. Bunsenges. Phys. Chem. 1957, 61, $962-$ 975.

[25]. Mataga, N.; Kaifu, Y.; Koizumi, M. Bull. Chem. Soc. Jpn. 1956, 29, 465470

[26]. Mataga, N.; Kubota, T. Molecular Interactions and Electronic Spectra, Dekker, New York, 1970

[27]. Bakshiev, N. G. Opt. Spektosk. (USSR) 1964, 16, 821-832.

[28]. Chamma, A.; Viallet, P. C. R. Acad. Sci. Paris, Ser. C 1970, 270, 19011904.

[29]. McRae, E. G. J. Phys. Chem. 1957, 61, 562-572.

[30]. Siddlingeshwar, B.; Hanagodimath, S. M.; Kirilova, E. M. J. Quant. Spectrosc. Radiat. Trans. 2011, 112, 448-456.

[31]. Reichardt, C. Solvents and Solvent Effects in Organic Chemistry, VCH, Weinheim, 1988.

[32]. Ravi, M.; Samanta, A.; Radhakrishnan, T. P. J. Phys. Chem. 1994, 98, 9133-9136.

[33]. Hasegawa, M.; Suzuki, Y.; Suzuki, F.; Nakaniski, H. J. Polym. Sci. A 1969, 7, 743-752

[34]. Ghazy, R.; Azim, S. A.; Shaheen, M.; El-Mekawey, F. Spectrochim. Acta A 2004, 60, 187-191.

[35]. Zachariasse, K. Private communication, $6^{\text {th }}$ International Conference on Solar Energy and Applied Photochemistry, "Solar'01", 3-8 April 2001, Cairo.

[36]. Diraison, M.; Millie, P.; Mialoq, Gustavsson, T. Chem. Phys. Letts. 1998, 282, 152-158.

[37]. McCarthy, P. K.; Blanchard, G. J. J. Phys. Chem. 1993, 97, 12205-12209.

[38]. Sharma, V. K.; Saharo, P. D.; Sharma, N.; Rastogi, R. C.; Mohan, D. Spectrochim. Acta A 2003, 59, 1161-1170.

[39]. DeMelo, J. S. S.; Becker, R. S.; Mocarita, A. I. J. Phys. Chem. 1994, 98, 6054-6058.

[40]. Gayathri, B. R.; Mannekutla, J. R.; Inamdar, S. R. J. Mol. Struct. 2008, 889, 383-393.

[41]. Cisse, L.; Djande, A.; Aaron, J. J. Spectrochim. Acta A 2011, 79, 428-436. 